\title{
Considérations sur l'épidémiologie dans un foyer hyperendémique de maladie du sommeil (T. gambiense) dans la région de Kasongo
}

\author{
par M. WERY et P. VAN WETTERE \\ Institut de Médecine Tropicale «Prince Léopold», B 2000 Anvers \\ Laboratoire de Parasitologie, c/o Université de Kinshasa, \\ B.P. 747, Kinshasa XI, Rép. du Zaïre
}

\begin{abstract}
Résumé
Les auteurs décrivent brièvement leurs observations épidémiologiques pour la trypanosomiase dans un biotope isolé constitué par un rassemblement artificiel de malades (léproserie). Les inconvénients de ce genre d'agglomérations sédentaires sont analysés.

La présence des trypanosomes a été recherchée chez 500 glossines non ténerales, en examinant toutes les portions du tube digestif et chez tous les habitants par la sérologie sur sang séché (immunofluorescence).
\end{abstract}

\section{Summary}

Epidemiology of trypanosomiasis is briefly analysed in an insulated biotope constituted by leprosy patients gathered in an artificially created village.

500 non teneral glossinae captured near the village were dissected and trypanosomes were searched in every part of the gut.

Indirect immunofluorescence test was carried out with dried blood samples of all the inhabitants of the village. 


\section{Description de la région.}

L'étude a porté sur deux villages distants l'un de l'autre de $5 \mathrm{~km}$ en zone d'endémie sommeilleuse, et qui présentent un tableau épidémiologique pour la trypanosomiase très dissemblable. Ils sont situés respectivement à 13 et $18 \mathrm{~km}$ au Nord-Ouest de Kasongo (Maniema, Rép. du Zaïre) dans un paysage de savane garni d'un réseau de forêts-galeries qui accompagnent les petits affluents du fleuve Lualaba.

Le premier de ces villages, artificiellement créé il y a plus de vingt ans pour héberger des lépreux, comporte 352 habitants dont la moitié environ sont en traitement pour la lèpre. Il a été construit sur le sommet d'une colline très évasée en contrebas de laquelle court une petite rivière accompagnée de son ruban d'arbres. Cette galerie forestière passe à environ 500 mètres au large du village. A son point le plus étroit, elle a une largeur de 50 mètres, mais de part et d'autre du village elle s'élargit considérablement pour former un bouquet forestier de forme irrégulière ayant une largeur maximale de un à deux kilomètres. Au-delà de ces étendues boisées, les arbres s'espacent et la galerie s'amenuise considérablement sans toutefois présenter de solution de continuité nette avec les galeries avoisinantes.

Le deuxième village (Kilongosi) est situé dans la savane ; plusieurs petites rivières avec galerie forestière clairsemée passent à proximité de ce village traditionnel installé là depuis très longtemps. Ce second village dont nous avons examiné la population a été choisi comme biotope témoin, car il est semblable aux autres petites agglomérations de la région.

\section{Observations entomologiques.}

a) Les glossines que nous avons eu l'occasion d'examiner ont été capturées uniquement sur l'homme, dans la forêt. Nous avons trouvé une grande majorité de $G$. fuscipes fuscipes et quelques spécimens de G. fusca sp.

b) Nous avons pu disséquer 500 glossines non ténerales provenant de la galerie forestière de la léproserie, afin de déterminer leur âge physiologique et le taux d'infection par Trypanosomes.

Dans les glandes salivaires, nous avons trouvé plus de $1 \%$ d'infection ( $T$. groupe brucei). En plus de ces infections salivaires, nous avons pu observer de nombreuses infections par $T$. congolense dont les hôtes vertébrés sont les chèvres et les moutons domestiques (diagnostics pratiqués par goutte épaisse colorée).

En fait, toutes les glossines disséquées qui étaient en âge d'être infectées (un mois ou plus) ont été trouvées porteuses de Trypanosomes.

c) L'homme est l'hôte préféré des tsé-tsés. Les échantillons capturés sur l'homme dans la forêt de la léproserie étaient constitués d'un haut pourcentage de femelles dont l'âge physiologique était élevé. Ce fait est contraire aux observations de l'un de nous (P. V. W.) au Bas-Congo, où le pourcentage de femelles de G. palpalis palpalis cap- 
turées sur l'homme est faible et l'âge physiologique nettement plus bas (Van wettere, sous presse).

Dans le village même de la léproserie, nous avons rencontré très peu de glossines. Le contact homme-glossine a lieu dans la forêt, où les villageois sont retenus une grande partie de la journée par des activités clandestines de distillation d'alcool. Ces installations sont soigneusement cachées et nous n'avons d'ailleurs pas réussi à y accéder. Les points d'eau ont peu d'importance vu la présence d'eau courante au village.

\section{Examen de la population.}

a) LA LÉPROSERIE.

Ce village comporte 352 habitants. Nous avons fait un prélèvement de sang séché sur papier filtre à tous les habitants. Au point de vue maladie du sommeil, il faut diviser les individus en deux groupes : d'une part ceux qui ont été diagnostiqués depuis 1968 , date de la reprise de la lutte contre la trypanosomiase dans cette région, et qui sont au nombre de 117 (33\%), tous avaient été traités ou étaient en cours de traitement au moment de notre passage ; d'autre part ceux qui, jusqu'à présent, n'avaient pas été dépistés par les équipes Fometro et qui étaient donc supposés indemnes de trypanosomiase $(66 \%)$.

Les résultats de la sérologie anti-Trypanosome par immunofluorescence (Wéry et al. $1970 a$ ) pour l'ensemble de la population sont les suivants: Dans le premier groupe (117 trypanosés traités depuis des périodes variables): réactions positives $(+++), 6,8 \%$; réactions douteuses, 14,5\% ; réactions négatives, 78,6\%. Dans le second groupe (sujets supposés indemnes) : réactions positives, $12,8 \%$; réactions douteuses, $2,5 \%$; réactions négatives, $84,7 \%$.

Notre expérience antérieure de cette réaction sérologique (Wéry et al. $1970 \mathrm{~b}$ ) nous permet de croire qu'au moins $80 \%$ des sujets présentant une réaction positive sont effectivement des trypanosés ( $T$. gambiense). On peut donc admettre que, dans le cas de la léproserie, plus de $9 \%$ (les trois quarts de $12,8 \%$ ) sont en incubation de trypanosomiase. Le dernier dépistage par les méthodes classiques effectué avant notre passage avait été pratiqué quatre mois plus tôt.

Ce pourcentage de nouveaux cas, que l'on peut évaluer à $18 \%$ par an, est très élevé et est en rapport avec le taux d'infection très élevé trouvé chez les glossines dans la galerie.

Il s'agit ici d'un biotope isolé où les mêmes glossines rencontrent les mêmes individus avec une grande fréquence.

\section{b) Village de Kilongosi.}

Ce village comporte 160 habitants dont $3,7 \%$ d'anciens cas traités et $96,3 \%$ de sujets supposés sains.

La sérologie nous a donné les résultats suivants: Parmi les anciens cas, aucune réaction positive, $33 \%$ de réactions douteuses et $67 \%$ de réactions négatives. Parmi 
les sujets sains, nous avons trouvé $2,5 \%$ de réactions positives, $4 \%$ de réactions douteuses et $93,5 \%$ de réactions négatives.

On retrouve ici un pourcentage de nouveaux cas normal pour une région d'endémie de trypanosomiase qui est sous contrôle d'une équipe mobile. Les habitants ne sont pas isolés et immobilisés dans leur village et ne dépendent pas d'une seule petite forêt. Leur contact avec les glossines est donc beaucoup plus diversifié.

\section{Moyens proposés pour remédier à la situation.}

a) La destruction des glossines par les insecticides après isolement complet de la galerie des autres gîtes à glossines se trouvant à proximité.

b) Traitement de tous les nouveaux malades (la sérologie peut aider à dépister les malades du sommeil en période d'incubation) puis pentamidinisation de tous les individus indemnes.

c) Déplacer la léproserie, ce qui est économiquement et psychologiquement peu recommandable.

d) Suppression des rassemblements artificiels de malades, dont les léproseries sont un exemple classique. Les conceptions nouvelles en matière de Santé publique ont amené à considérer les lépreux comme des malades non différents des autres qui peuvent être traités en ambulatoire et vivre parmi leurs concitoyens au village. On pourrait donc renvoyer les lépreux chez eux après un dépistage soigneux de tous les trypanosés.

\section{Bibliographie}

Van Wettere (P.), 1971. - Bijdrage tot de kennis van de biologie van Glossina palpalis palpalis en de faktoren die de overdracht van $T$. gambiense beinvloeden. Sous presse.

Wéry (M.), Wéry Paskoff (S.) and VAN Wettere (P.), 1970 (a). - The diagnosis of human african trypanosomiasis ( $T$. gambiense) by the use of the fluorescent antibody test. 1. Standardisation of an easy technique to be used in mass surveys. Ann. Soc. belge Méd. trop., 50, 613-634.

-, Van Wettere (P.), Wéry Paskoff (S.), Van Meirvenne (N.) and Mesatewa (M.), 1970 (b). - The diagnosis of human african trypanosomiasis (T. gambiense) by the use of the fluorescent antibody test. 2. First results of field application. Ann. Soc. belge Méd. trop., 50, 711-730. 